\title{
Cambios en la población de protozoarios ruminales por efecto de la inclusión de Canavalia ensiformis a la dieta de bovinos.
}

\author{
Artículo Original
}

Carlos A. Sandoval-Castro, Fernando Herrera y Gómez.

Departamento de Nutrición Animal, Facultad de Medicina Veterinaria y Zootecnia, Universidad Autónoma de Yucatán, Mérida, Yucatán, México.

\section{RESUMEN.}

Introducción. Canavalia ensiformis es una leguminosa que destaca por su contenido de nutrimentos. Sin embargo, posee numerosos compuestos tóxicos que pueden limitar su utilización. Se ha reportado efecto de estos compuestos in vitro sobre fauna ruminal, pero se desconoce su efecto tóxico in vivo.

Objetivo. El objetivo del presente trabajo fue analizar los cambios en la población de protozoarios ruminales como respuesta al consumo de canavalia.

Materiales y Métodos. Se emplearon cuatro bovinos con un peso de $264.5 \mathrm{Kg}$ en un diseño doble conmutativo con arreglo factorial $2 \times 2$. Los animales fueron alimentados con pasto Taiwán ad libitum y canavalia o sorgo como suplemento (c. $27 \%$ de la dieta en base seca). Se colectó líquido ruminal después de tres semanas de adaptación a la dieta y se contaron las poblaciones de protozoarios ruminales. Los datos normalizados utilizando su $\log _{10}$ y fueron analizados mediante un modelo lineal general. Las medias fueron comparadas mediante la prueba de mínima diferencia significativa.

Resultados. Se observó un incremento $(p<0.05)$ en los conteos de protozoarios flagelados en las dietas que contenían canavalia $\left(10^{6.345}\right.$ vs $\left.10^{5.247}\right)$, pero los holotricos y entodinomorfos disminuyeron $\left(10^{3.717}\right.$ vs $10^{4.364}$ y $10^{4.407}$ vs $10^{4.933}$ respectivamente $\mathrm{p}<0.05)$. Las cuentas totales de protozoarios fueron mayores en dietas con canavalia si el total incluía flagelados $\left(10^{6.369} \mathrm{vs}\right.$ $\left.10^{5.575}\right)$ y menores si los excluía $\left(10^{4.505}\right.$ vs $\left.10^{5.093}\right)$ $(\mathrm{p}<0.05)$.

Discusión. Los cambios en las diferentes poblaciones de protozoarios son una respuesta de adaptación al consumo de canavalia. Los diferentes grupos de protozoarios ruminales parecen tener diferente respuesta y/o capacidad de adaptación a los tóxicos de la canavalia.

(Rev Biomed 2001; 12:166-171)

Solicitud de sobretiros: Carlos A. Sandoval-Castro, Departamento de Nutrición Animal, Facultad de Medicina Veterinaria y Zootecnia, Universidad Autónoma de Yucatán, Apdo Postal 4-116 Itzimná, C.P. 97100, Mérida, Yucatán, México. Fax: +52 (9) 9423205 Email: c.a.sandoval@excite.com Recibido el 19/Junio/2000. Aceptado para publicación el 29/Septiembre/2000. 


\section{CA Sandoval-Castro, F Herrera y Gómez.}

Palabras clave: Canavalia ensiformis, protozoarios ruminales, bovinos.

\section{SUMMARY.}

Changes on rumen protozooa population induced by the inclusión of Canavalia ensiformis in cattle diets.

Introduction. Canavalia ensiformis is a legume which stands out for its nutritional value. However, it also has several toxic compounds that limits its use. In vitro toxic effect of canavalia upon rumen protozoa has been reported but its in vivo toxicity is unknown.

Objective. The objective of the present workt was to assess the changes of the rumen protozoa population when cattle are fed canavalia in their diet.

Material and Methods. Four bulls (264.5 Kg live weight) fitted with rumen cannulae were used in a $2 \times 2$ crossover design with factorial arrangement. The animals were fed ad libitum with taiwan grass hay and canavalia or sorghum grain as a supplement (c. $27 \%$ of diet on dried mead base). Rumen liquour was collected after a 3-week adaptation period and the rumen protozoa were classified and counted. Data was analyzed after $\log _{10}$ transformation using a GLM procedure. Means were compared by the LSD test.

Results. An increment was observed $(\mathrm{p}<0.05)$ in the flagella population when the diets included canavalia $\left(10^{6.345}\right.$ vs $\left.10^{5.247}\right)$, but holotrics y entodinia were lowered $\left(10^{3.717}\right.$ vs $10^{4.364}$ y $10^{4.407}$ vs $10^{4.933}$ respectively $\mathrm{p}<0.05)$. The total protozoa numbers were higher with canavalia if flagella were included $\left(10^{6.369}\right.$ vs $\left.10^{5.575}\right)$ and lower if flagella were excluded $\left(10^{4.505}\right.$ vs $\left.10^{5.093}\right)(\mathrm{p}<0.05)$.

Discussion. Changes in the protozoa population were an adaptative response to canavalia intake. Different protozoa groups seem to have different abilities to adapt/ and/or respond to the canavalia toxics.

(Rev Biomed 2001; 12:166-171)
Key words: Canavalia ensiformis, rumen protozoa, cattle.

\section{INTRODUCCIÓN.}

Canavalia ensiformis es una leguminosa que destaca por su elevado valor nutricio, especialmente por su contenido de proteínas, por lo que ha sido empleada como una fuente proteica en la alimentación tanto de monogástricos como de rumiantes $(1,2)$. Sin embargo, la canavalia posee numerosos factores antinutricios que pueden limitar su uso principalmente en monogástricos, por lo que numerosos trabajos se han enfocado en su detoxificación, con resultados diversos (2).

La incorporación de canavalia en dietas para rumiantes ha originado respuestas variadas; algunos autores han encontrado reducción en el consumo y digestibilidad de la dieta (3-5), mientras que otros no han encontrado efecto adverso (6-8) y han recomendado su uso en la alimentación de rumiantes como suplemento proteínico y/o energético.

Dada la función fermentativa del rumen, ha sido posible utilizar el grano de canavalia crudo como suplemento obteniéndose buenos resultados (6-8). Sin embargo, se ha mencionado que la inclusión de canavalia tiene un posible efecto sobre las bacterias ruminales (9) lo que causaría la depresión de la digestión observado en algunos reportes. La magnitud del efecto ha sido minimizado (10), y se ha encontrado que poblaciones mixtas de bacterias ruminales son capaces de adaptarse después de varias semanas (9). La inclusión de canavalia en la dieta parece no afectar el número de protozoarios viables, excepto por una ligera disminución de la población de entodinium (11). Sin embargo, dado que los protozoarios ruminales pueden contribuir hasta con el 34\% de la digestión de la fibra (12), es importante efectuar estudios sobre cambios en su población debido a la inclusión de canavalia en la dieta y elucidar su posible toxicidad para esta población y de esta manera conocer si estos cambios son los

\section{Revista Biomédica}


Canavalia ensiformis y protozoarios ruminales.

responsables de las reducciones encontradas en la digestibilidad de la fibra a nivel ruminal y/o de los proceso de adaptación a los diversos compuestos contenidos en la canavalia. Así, el objetivo del presente trabajo fue conocer los cambios que ocurren en la población de protozoarios ruminales en animales consumiendo canavalia como parte de su dieta.

\section{MATERIALES Y MÉTODOS.}

Animales. Se emplearon 4 novillos de raza cebú, provistos de cánula ruminal, con un peso promedio de $264.5 \pm 12.6 \mathrm{Kg}$. Los animales se alojaron en corrales individuales con piso de cemento y techos de lámina de zinc.

Adaptación. Los animales fueron adaptados paulatinamente a la dieta cambiando la alimentación anterior a la experimental en el transcurso de una semana, posteriormente se permitió un periodo de adaptación adicional de tres semanas.

Alimentación. Los animales fueron mantenidos bajo las dietas presentadas en el cuadro 1 , siendo los tratamientos experimentales la inclusión o no de canavalia picada en la dieta. Las dietas fueron balanceadas para proporcionar una cantidad de energía metabolizable igual a 1.25 veces las necesidades de mantenimiento (13), los aportes nutrimentales de los diferentes ingredientes son presentados en el cuadro 2.

Ambas dietas (control y experimental)
Cuadro 1

Composición de las dietas de los animales durante el experimento (KgMS/día).

\begin{tabular}{lcccc}
\hline \multicolumn{5}{c}{ Animales } \\
& $\mathrm{A}$ & $\mathrm{B}$ & $\mathrm{C}$ & $\mathrm{D}$ \\
\hline 1er. Periodo & & & & \\
Heno Taiwán & 2.73 & 3.66 & 2.73 & 3.44 \\
Melaza & 0.36 & 0.48 & 0.36 & 0.45 \\
Canavalia & 1.17 & 0 & 1.17 & 0 \\
Sorgo & 0 & 0.85 & 0 & 0.80 \\
2o. Periodo & & & & \\
Heno Taiwán & 3.25 & 3.08 & 3.33 & 2.90 \\
Melaza & 0.37 & 0.35 & 0.37 & 0.33 \\
Canavalia & 0 & 1.26 & 0 & 1.19 \\
Sorgo & 0.74 & 0 & 0.76 & 0 \\
\hline
\end{tabular}

$\mathrm{MS}=$ Materia seca

incluyeron melaza para estimular el consumo total del suplemento. La inclusión de sorgo en la dieta control fue con el objeto de proporcionar cantidades similares de energía en ambas dietas. Sorgo y melaza también fueron utilizados en ambas dietas para evitar el efecto confundido de cambios en la población de protozoarios por el aporte de azucares y almidones a nivel ruminal (cuadro 1).

El alimento fue proporcionado diariamente a las 07:30 horas, previo pesaje del rechazo de heno de pasto taiwan (Pennisetum purpureum) del día anterior. Se evitó el rechazo de la canavalia ofrecida mediante la infusión del remanente del mismo que no hubiera sido consumido a las 11:30 horas a través de la fístula ruminal (cuando existiese rechazo) y se les proporcionó agua ad libitum en

Cuadro 2

Composición química (\%) de los componentes de las dietas.

\begin{tabular}{lllllllll}
\hline & \multicolumn{3}{c}{ Primer Periodo } & \multicolumn{7}{c}{ Segundo Periodo } \\
& MS & PC & MO & EM* & MS & PC & MO & EM \\
\hline Sorgo & 88.64 & 9.24 & 98.43 & 3.51 & 87.06 & 10.50 & 97.87 & 3.49 \\
Heno Taiwán & 89.89 & 6.46 & 92.91 & 1.82 & 89.16 & 6.67 & 92.54 & 1.83 \\
Canavalia & 87.99 & 29.19 & 96.47 & 3.46 & 84.93 & 23.50 & 97.18 & 3.47 \\
Melaza & 65.21 & 3.36 & 90.98 & 3.24 & 75.63 & 3.75 & 91.92 & 3.28 \\
\hline
\end{tabular}

MS = Materia Seca, $\mathrm{PC}=$ Proteína Cruda, $\mathrm{MO}=$ Materia Orgánica, $\mathrm{EM}^{*}=$ Energía metabolizable $(\mathrm{MCal} / \mathrm{Kg} \mathrm{MS})$

Vol. 12/No. 3/Julio-Septiembre, 2001 


\section{CA Sandoval-Castro, F Herrera y Gómez.}

recipientes de plástico.

Muestreo de líquido ruminal. Dada la variación diurna normal de las poblaciones de protozoarios se tomaron muestras de líquido ruminal a las 0 , 1.5, 3, 6, 9 y 12 horas posteriores a la alimentación (7:30 AM) utilizando un sistema de vacío rústico. Se colectó en cada muestra un total de $200 \mathrm{~mL}$, los cuales fueron filtrados a través de una gasa de algodón para reducir la cantidad de residuos de alimento conservando $20 \mathrm{~mL}$. Posteriormente el líquido ruminal fue preservado con fenol (2 gotas por muestra) y conservado en refrigeración hasta el conteo de protozoarios, el cual se realizó empleando una cámara Fucsh-Rosenthal. La preparación de la muestra para conteo y tinción de los protozoarios con MFS (solución salina-verde de metileno-formalina) fue realizada de acuerdo a Ogimoto e Imai (14).

\section{Diseño experimental.}

Dos animales fueron mantenidos en la dieta con canavalia y dos animales fueron suplementados con sorgo en el primer periodo (cuadro 1). Al iniciar el segundo periodos los animales que durante el primer periodo fueron mantenidos en la dieta de canavalia cambiaron a la dieta con sorgo y aquellos alimentados con sorgo cambiaron a la dieta con canavalia. Se consideró a las muestras de la población ruminal de protozoarios en un ambiente normal aquellas que fueron obtenidas en los animales durante el periodo de suplementación con sorgo. Los cambios en la población fueron observados en aquellos animales consumiendo canavalia. Todos los animales fueron empleados en ambas dietas al cabo de los 2 periodos experimentales.

El diseño experimental empleado correspondió al doble conmutativo 2 × 2 con arreglo factorial, siendo la variable principal la inclusión o no de canavalia en la dieta.

Las observaciones de concentración de protozoarios fueron normalizadas transformandolas a sus logaritmos ${ }_{10}$, posteriormente fueron analizadas mediante un análisis de varianza a través del paquete estadístico Minitab 12 (15), empleando el procedimiento general de modelos lineales (GLM). La diferencia entre las diversas poblaciones de protozoarios se analizó mediante la prueba de diferencia mínima significativa.

\section{RESULTADOS.}

Durante el desarrollo del experimento se observó una población que fue clasificada como protozoarios flagelados por sus características morfológicas y aún cuando la tinción MFS no permite una clara distinción entre zoosporas y flagelados. Se descartó que pudieran ser zoosporas de hongos ruminales debido a que se observo numerosos individuos en etapa de reproducción por división asexual (15). No obstante, las cuentas totales de protozoarios fueron analizadas con y sin la inclusión de esta población. El cuadro 3 muestra las poblaciones de protozoarios encontradas durante la prueba. Se pudo observar que la población de los protozoarios flagelados, fue significativamente mayor $(\mathrm{P}<0.05)$ en la dieta que incluía canavalia. Sin embargo se observó que las poblaciones de holotricos y entodinomorfos fueron mayores en los animales cuya dieta no contenía canavalia $(p<0.05)$. Las poblaciones totales fueron mayores en las dietas con canavalia si el total incluye la población de flagelados $(\mathrm{p}<0.05)$ y menores si estos eran excluidos $(\mathrm{p}<0.05)$.

\section{DISCUSIÓN.}

Se ha mencionado que los tóxicos de canavalia tienen un efecto sobre la población de protozoarios ruminales, ya que se ha encontrado que en condiciones in vitro, cuando se agrega Concanavalina A en medios de cultivos existe una muerte de protozoarios como signo indicador de su actividad. Canavanina, por otro lado, no ha demostrado tener un efecto significativo sobre las poblaciones totales de protozoarios viables in vitro, excepto por una ligera disminución de las

\section{Revista Biomédica}




\section{Canavalia ensiformis y protozoarios ruminales.}

Cuadro 3

Población de protozoarios por clase y totales $\left(\log _{10}\right.$ protozoarios / $\mathrm{ml}$ líquido ruminal) encontrados en rumen de bovinos consumiendo Canavalia ensiformis (cc) o sorgo (sc) como suplemento principal en la dieta.

\begin{tabular}{lcccccccccc}
\hline & \multicolumn{2}{c}{ Holotricos } & \multicolumn{2}{c}{ Entodinios } & \multicolumn{2}{c}{ Flagelados } & \multicolumn{2}{c}{ Total c Fla } & \multicolumn{2}{c}{ Total s Fla } \\
Hora & $\mathrm{cc}$ & $\mathrm{sc}$ & $\mathrm{cc}$ & $\mathrm{sc}$ & $\mathrm{cc}$ & $\mathrm{sc}$ & $\mathrm{cc}$ & $\mathrm{sc}$ & $\mathrm{cc}$ & $\mathrm{sc}$ \\
\hline 0 & $3.767 \mathrm{a}$ & $4.407 \mathrm{~b}$ & $4.450 \mathrm{a}$ & $5.070 \mathrm{~b}$ & $6.346 \mathrm{a}$ & $5.433 \mathrm{~b}$ & $6.375 \mathrm{a}$ & $5.647 \mathrm{~b}$ & $4.536 \mathrm{a}$ & $5.184 \mathrm{~b}$ \\
1.5 & $3.605 \mathrm{a}$ & $4.358 \mathrm{~b}$ & $4.324 \mathrm{a}$ & $4.981 \mathrm{~b}$ & $6.391 \mathrm{a}$ & $5.060 \mathrm{~b}$ & $6.409 \mathrm{a}$ & $5.589 \mathrm{~b}$ & $4.406 \mathrm{a}$ & $5.079 \mathrm{~b}$ \\
3 & $3.499 \mathrm{a}$ & $4.635 \mathrm{~b}$ & $4.354 \mathrm{a}$ & $4.664 \mathrm{a}$ & $6.470 \mathrm{a}$ & $5.692 \mathrm{a}$ & $6.479 \mathrm{a}$ & $5.832 \mathrm{~b}$ & $4.418 \mathrm{a}$ & $5.138 \mathrm{~b}$ \\
6 & $3.610 \mathrm{a}$ & $4.188 \mathrm{~b}$ & $4.304 \mathrm{a}$ & $4.880 \mathrm{~b}$ & $6.246 \mathrm{a}$ & $4.905 \mathrm{~b}$ & $6.262 \mathrm{a}$ & $5.349 \mathrm{~b}$ & $4.391 \mathrm{a}$ & $4.985 \mathrm{~b}$ \\
9 & $3.841 \mathrm{a}$ & $4.256 \mathrm{a}$ & $4.365 \mathrm{a}$ & $4.959 \mathrm{~b}$ & $6.183 \mathrm{a}$ & $5.328 \mathrm{~b}$ & $6.217 \mathrm{a}$ & $5.575 \mathrm{~b}$ & $4.523 \mathrm{a}$ & $5.046 \mathrm{~b}$ \\
12 & $3.979 \mathrm{a}$ & $4.341 \mathrm{a}$ & $4.648 \mathrm{a}$ & $5.047 \mathrm{~b}$ & $6.434 \mathrm{a}$ & $5.062 \mathrm{~b}$ & $6.473 \mathrm{a}$ & $5.456 \mathrm{~b}$ & $4.756 \mathrm{a}$ & $5.129 \mathrm{~b}$ \\
$\mathrm{EE} \overline{\mathrm{x}}$ & 0.1761 & 0.1084 & 0.2754 & 0.1549 & 0.1197 \\
\hline$\overline{\mathrm{x}}$ general & $3.717 \mathrm{a}$ & $4.364 \mathrm{~b}$ & $4.407 \mathrm{a}$ & $4.933 \mathrm{~b}$ & $6.345 \mathrm{a}$ & $5.247 \mathrm{~b}$ & $6.369 \mathrm{a}$ & $5.575 \mathrm{~b}$ & $4.505 \mathrm{a}$ & $5.093 \mathrm{~b}$ \\
EE $\overline{\mathrm{x}}$ & 0.0719 & 0.0443 & 0.1124 & 0.0632 & \multicolumn{2}{c}{0.0489} \\
\hline
\end{tabular}

La dieta basal en ambos casos consistió en heno de pasto taiwan + melaza como saborizante del suplemento - (canavalia o sorgo)

Entodinios $=$ Entodinomorfos, Total $\mathrm{c}$ fla $=$ total de protozoarios incluyendo a los Flagelados, Total $\mathrm{s}$ fla $=$ total de protozoarios excluyendo a los flagelados. Medias dentro de hilera (para una misma población) con letras diferentes difieren a $\mathrm{p}<0.05$.

poblaciones de Entodinium (11). Sin embargo, el efecto real de estas sustancias in vivo es todavía desconocido.

Concordando con la evidencia de posible toxicidad, en el presente experimento, se observo una disminución $(\mathrm{p}<0.05)$ de la población de Entodinomorfos en la dieta que incluía canavalia. Los animales del presente experimento consumieron un equivalente a $6.9 \times 10^{-3} \mathrm{~mol}$ canavanina/L de licor ruminal, cantidad ligeramente inferior a la reportada como ligeramente tóxica para entodinios por Mora y col. (11) de $0.85 \mathrm{~mol} / \mathrm{L}$. No obstante, la diferencia entre las poblaciones de Holotricos, Entodinomorfos y flagelados entre dietas fue significativa $(p<0.05)$, encontrándose mayores cantidades de los primeros en dietas sin canavalia, mientras que grandes cantidades de flagelados fueron encontrados en dietas con canavalia.

Se ha reportado que el follaje de algunas especies de árboles como Sapindus saponaria y Enterolobium ciclocarpum pueden tener propiedades defaunantes in vitro e in vivo (16). Sin embargo la respuesta es de magnitud diferente, ya que las concentraciones tóxicas en condiciones in vitro no alcanzan a defaunar in vivo, aunque si reducen grandemente la población de protozoarios totales (16). Respuestas de este tipo no son extrañas dado el ambiente dinámico del rumen donde la tasa de recambio y constante flujo de materiales al tracto posterior reduce su tiempo de contacto con los microorganismo ruminales y por lo tanto su posible efecto tóxico.

Por otro lado, Rosales y col. (17) reportan cambios en la composición relativa de las especies de protozoarios ruminales como una respuesta adaptativa al consumo de Erythrina poeppigiana. Es probable que una respuesta de este tipo sea la causa de los cambios observados en bovinos alimentados con canavalia, ya que se observó un incremento de flagelados (clase no común de protozoario ruminal) y un aumento neto de la población de protozoarios cuando la cuenta total incluía flagelados.

Posiblemente este tipo de respuesta es producto de procesos de adaptación a la dieta, ya que los flagelados se presentaron en mayor número en los animales consumiendo canavalia $(\mathrm{p}<0.01)$, 


\section{CA Sandoval-Castro, F Herrera y Gómez.}

y su número difiriere significativamente por efecto de dieta $(\mathrm{p}<0.01)$. No se encontraron reportes semejantes en la literatura del efecto de canavalia sobre fauna ruminal. Las causas y mecanismos de esta adaptación deberán ser estudiadas con mayor detalle dado que es probable que esta fauna pudiera ser parcialmente responsable de los mecanismos de adaptación y detoxificación de los compuestos antinutricios contenidos en la canavalia.

\section{REFERENCIAS.}

1.- Belmar R, Ellis N. El efecto de diferentes tratamientos sobre el valor nutritivo de las semillas de canavalia ensiformis. En: Alternativas y valor nutritivo de algunos recursos alimenticios destinados a producción. Informe Provisional No. 16. Fundación Internacional para la Ciencia; 1985. p. 139-46.

2.- Belmar R, Nava-Montero R, Sandoval-Castro C, McNab JM. Jack bean (Canavalia ensiformis L. DC) in poultry diets: Antinutritional factors and detoxification studies- a review. World's Poultry Sci J 1999; 55:37-59.

3.- Camacho D, Escobar A, Parra R. Efecto del tamaño de partículas del pericarpio y del nivel de grano de canavalia ensiformis sobre el consumo y la digestibilidad. Informe anual IPA 1985/1986. Universidad Central de Venezuela;1987. p. 41-2.

4.- González A, Escobar A. Uso de la canavalia en becerros predestete. II. Alimentación a base de planta integral de yuca. Informe anual IPA 1987. Universidad Central de Venezuela; 1989. p. 44-5.

5.- Paredes L, Escobar A, Fernandez L. Efecto del nivel de suplementación con canavalia sobre el crecimiento de becerros. Informe anual IPA 1985/1986. Universidad Central de Venezuela. 1987; p. 34-5.

6.- Mora M, Combellas J, Parra O. Complementariedad de sustratos entre harina de grano de canavalia y urea. Informe anual IPA 1988/1989. Universidad Central de Venezuela; 1990. p. 51-2.

7.- Váquez W, Silva EJ, Combellas J, Escobar A. Evaluación de la harina de grano de canavalia y harina de pescado como suplementos proteicos en raciones para becerros postdestete. Informe anual IPA 1985/1986. Universidad Central de Venezuela; 1987. p. 36-7.
8.- Silva EJ, Vásquez W, Combellas J, Escobar A. Comparación de dos formas de suministro de grano de canavalia con y sin suplementación de harina de canavalia con y sin suplementación de harina de pescado en raciones para becerros. Informe anual IPA 1985/1986. Universidad Central de Venezuela; 1987. p. 38-9.

9.- Domínguez-Bello MG, Stewart C. Effects of feeding Canavalia ensiformis on the rumen flora of sheep, and of the toxic amino acid canavanine on rumen bacteria. System Appl Microbiol 1990; 13: 388-93.

10.- Herrera F, Godoy R. El uso de la Canavalia ensiformis en la alimentación de rumiantes. En: Memorias 1a. Reunión sobre Producción Animal Tropical. Cárdenas, Tabasco, Julio. CEICAFAS/FMVZ-UADY/Col. Postgraduados. 1989. S/p.

11.- Mora M, Domínguez MG, Escobar A. Uso de la canavalia ensiformis en la alimentación de rumiantes. En: Seminario-taller de trabajo sobre Canavalia ensiformis. Maracay Venezuela. 1991. S/p.

12.- Demeyer DI. Rumen microbes and digestion of plant cell walls. Agric Environ 1981; 6:295-337

13.- ARC (Agricultural Research Coulcil). The nutrient requirements of ruminant livestock. Farnham Royal, Slough U.K.: Commonwealth Agricultural Bureaux; 1980.

14.- Ogimoto K, Imai S. Atlas of rumen microbiology. Tokyo:Japan Scientific Societies Press; 1981.

15.- Minitab, statistical software release 12. Minitab Inc. State College, PA. USA. 1998.

16.- Díaz A, Avendaño M, Escobar A. Use of tropical plants as defaunating agents and its effects on animal metabolism. En: Dual purpose cattle research. Anderson S, Wadsworth $\mathrm{J}$, editores. Proceedings of an international workshop, March 1992, Mérida, México. IFS/FMVZ-UADY; 1995. p. $273-80$.

17.- Rosales M, Laredo M, Cuesta A, Anzola H, Hernández L. The use of forage tree leaves to control protozoa population in the rumen. En: Dual purpose cattle research. Anderson S, Wadsworth J, editores. Proceedings of an international workshop, March 1992, Mérida, México. IFS/ FMVZ-UADY; 1995. p. 281-8.

\section{Revista Biomédica}

